\title{
(2) OPEN ACCESS \\ A stakeholder meeting exploring the ethical perspectives of immediately sequential bilateral cataract surgery
}

\author{
Matthew Quinn 이, ${ }^{1}$ Daniel Gray, ${ }^{2}$ Ahmed Shalaby Bardan, ${ }^{3}$ \\ Mehran Zarei-Ghanavati 다, ${ }^{4}$ John Sparrow, ${ }_{1}^{5}$ Christopher Liu

\begin{abstract}
- Additional material is published online only. To view, please visit the journal online (http://dx.doi.org/10.1136/ medethics-2020-106412).

${ }^{1}$ Royal United Hospital Bath NHS Trust, Bath and North East Somerset, UK

${ }^{2}$ School of Social Sciences, Cardiff University, Cardiff, UK ${ }^{3}$ Sussex Eye Hospital, Brighton and Sussex University Hospitals NHS Trust, Brighton, UK

${ }^{4}$ Farabi Eye Hospital, Tehran University of Medical Sciences, Tehran, The Islamic Republic of Iran

${ }^{5}$ Bristol Eye Hospital, University Hospitals Bristol NHS Trust, Bristol, UK

${ }^{6}$ Brighton and Sussex Medical School, Brighton, United Kingdom

${ }^{7}$ Tongdean Eye Clinic, Hove, United Kingdom
\end{abstract}

\section{Correspondence to}

Professor Christopher Liu, Sussex Eye Hospital, Brighton BN2 5BF, UK; cscliu@aol.com

Received 7 May 2020 Revised 22 July 2020 Accepted 28 July 2020

\section{Check for updates}

(C) Author(s) (or their employer(s)) 2020. Re-use permitted under CC BY-NC. No commercial re-use. See rights and permissions. Published by BMJ.

To cite: Quinn M, Gray D, Bardan AS, et al. J Med Ethics Epub ahead of print: [please include Day Month Year]. doi:10.1136/

medethics-2020-106412

\section{ABSTRACT \\ Purpose The purported benefits and risks of} immediately sequential bilateral cataract surgery (ISBCS) have been well described, yet the procedure remains controversial among UK ophthalmologists. As many of the controversies of ISBCS are underpinned by ethical dilemmas, the aim of this work was to explore the ethical perspectives of ISBCS from a variety of stakeholder viewpoints.

Method A semi-structured independent stakeholder meeting was convened at the Royal College of Ophthalmologists London headquarters in June 2018. In total, 29 stakeholders attended the meeting. The professional characteristics of stakeholders included but were not limited to: ophthalmologists (9), patients (5), religious leaders (4), ethicists (2), lawyers (2) and commissioners (1). Thematic qualitative analysis using methodology proposed by Braun and Clarke was conducted on the resultant transcript of the discussion. Results Themes identified include: (1) beneficence and non-maleficence (patient benefits, patient risks, the uncertainties of risk, patient interpretation of the riskbenefit analysis); (2) autonomy (informed consent, the barriers to communication); (3) distributive justice (the allocation of resources: the individual vs the collective). Conclusion This analysis provides a reference point for the ethical factors surrounding ISBCS. The stakeholders concluded that this approach was an ethical undertaking provided patient autonomy was appropriately attained. This requires a patient's interpretation of the risk-benefit balance, which must include an understanding of the low but unquantifiable risk of severe complications. A surgeon must aim to minimise risks through the adaption of accepted surgical protocols and by performing appropriate patient selection. Currently, cost savings to healthcare that may occur following the implementation of ISBCS should be considered a secondary benefit of the protocol.

\section{INTRODUCTION}

Epidemiological models based on ageing demographics estimate cataract surgery numbers to increase by $50 \%$ by 2035 . Literature from the Royal College of Ophthalmologists (RCOphth) has reflected concern over the future demand on UK ophthalmologists. ${ }^{1}$ New surgical efficiencies have therefore been sought to increase cataract productivity while maintaining excellent outcomes. ${ }^{12}$ Recently, a modified approach to the standard cataract surgery pathway, known as immediately sequential bilateral cataract surgery (ISBCS) has been attracting interest. This procedure consists of operating on both symptomatic eyes at the same sitting. $^{23}$

The purported benefits of ISBCS have been well described in the literature, these include both direct patient and wider economic benefits. ${ }^{2}{ }^{3}$ Many proposed risks of ISBCS are focused on the potential for bilateral complications, with bilateral endophthalmitis often cited as the primary concern for surgeons. ${ }^{34}$ Within the UK, ISBCS remains a controversial procedure and uptake by RCOphth members is low $(13.9 \%))^{5}$ In 73 centres where at least one patient had undergone ISBCS, the overall rate was $0.4 \%$ of the cataract operations performed. ${ }^{6}$ Some commentators believe that for a routine elective procedure, ISBCS should not be undertaken as the potential benefits do not outweigh the potential harms of the protocol. ${ }^{7}$

The principle of not inflicting harm (nonmaleficence) and promoting good (beneficence) form an integral basis for many theoretical approaches in medical ethics. In 1979, American Bioethicists Beauchamp and Childress proposed that ethical decision making required the consideration of wider aspects of equity and fairness (justice) and respect for an individual's free will (autonomy). ${ }^{8}$ Whereas Beauchamp and Childress described the 'classical' principles of medical ethics, this represents only one approach within ethical decision making. ${ }^{9}$ The utility of ethical debate exists in individuals balancing and weighing ethical considerations within context-specific scenarios. Individuals may also use the application of various personal moral standards to arrive at different conclusions. $^{8} 10$

Ethical considerations are often taken into account when implementing novel techniques into practice. ${ }^{1112}$ As many of the controversies of ISBCS are underpinned by ethical factors, the aim of this work was to explore the ethical perspectives of ISBCS from a variety of stakeholder viewpoints. Additionally, it is our hope that the representation of a variety of expert and patient perspectives will guide the identification of future areas of research for ISBCS.

\section{METHOD}

\section{Stakeholder meeting design and setting}

For this independent meeting, we have defined stakeholders as: persons who may be directly or indirectly affected by a change in the protocol for 


\begin{tabular}{|c|c|}
\hline Professional capacity & Number of attendees \\
\hline \multicolumn{2}{|l|}{ Healthcare professionals } \\
\hline Ophthalmologist & 6 \\
\hline Ophthalmologist and ethicist & 1 \\
\hline Ophthalmologist of Muslim faith & 1 \\
\hline Ophthalmologist and public health ophthalmologist & 1 \\
\hline Ophthalmic nurse and patient & 3 \\
\hline Optometrist & 1 \\
\hline \multicolumn{2}{|l|}{ Other professionals } \\
\hline Bioethicist & 1 \\
\hline Bioethicist and political philosopher & 1 \\
\hline Commissioner & 1 \\
\hline Lawyer & 2 \\
\hline Health economist & 1 \\
\hline \multicolumn{2}{|l|}{ Religious persons } \\
\hline Catholic priest & 1 \\
\hline Academic of Jewish faith & 1 \\
\hline Muslim chaplain and scholar & 1 \\
\hline Rabbi & 1 \\
\hline \multicolumn{2}{|l|}{ Lay attendees } \\
\hline Lay trustee of the RCOphth & 1 \\
\hline Lay member of the RCOphth & 1 \\
\hline Medical student & 1 \\
\hline Patient & 2 \\
\hline Patient advocate & 1 \\
\hline Total & 29 \\
\hline
\end{tabular}

RCOphth, Royal College of Ophthalmologists.

delivery of cataract surgery. The stakeholders selected participated as a collective group of non-author contributors, to assist in the identification of their ethical perspectives of ISBCS. Questions were formulated and distributed to stakeholders in advance following confirmation of attendance (online supplementary material). The questions provided were intended to elicit discussion of ethical topics, but not to provide the basis for analysis. The content of the questions was validated by three senior ophthalmologists, each with divergent views on ISBCS.

The meeting took place at the RCOphth London headquarters in June 2018. The meeting was semi-structured with discussions facilitated by an independent chair. All viewpoints represented were personal views and not necessarily representative of each stakeholder's respective organisation. The event was audiovisually recorded with consent from participants to publish the content in the public domain. ${ }^{1314}$

\section{Stakeholders}

Stakeholders were invited via email through purposive sampling. Where individual stakeholder's views of ISBCS were known a priori, attempts were made to select a mixture of participants both for and against the procedure. A total of 29 stakeholders participated. Key stakeholder groups included but were not limited to: ophthalmologists (9), patients (5), nurses (2), ethicists (2), lawyers (2), religious persons (4), optometrists (1), commissioners (1) and health economists (1). A full list of the primary professional capacities of the attendees is displayed in table 1 .
Table 2 Themes and subthemes identified at the stakeholder meeting

\begin{tabular}{ll}
\hline Theme & Subtheme \\
\hline 1. Beneficence and non-maleficence & $\begin{array}{l}1.1 \text { Patient benefits } \\
1.2 \text { Patient risks }\end{array}$ \\
& $\begin{array}{l}1.3 \text { The uncertainty of risk } \\
1.4 \text { Patient interpretation of the risk-benefit } \\
\text { analysis }\end{array}$ \\
\hline $\begin{array}{l}2.1 \text { Informed consent } \\
\text { 2. Autonomy }\end{array}$ & $\begin{array}{l}2.2 \text { The barriers to communication } \\
\text { 3.1 The allocation of resources: the individual } \\
\text { versus the collective }\end{array}$ \\
\hline
\end{tabular}

\section{Data analysis}

The data were transcribed using intelligent verbatim and imported into qualitative software organiser EnVivo12. The analysis was conducted using a 'bottom up' or inductive approach, using an open coding technique in which the themes developed were data driven. The approach to thematic analysis was conducted based on described protocol by Braune and Clarke. ${ }^{15}$ For validation purposes, the entire set of data was then re-coded independently by a second qualitative researcher (DG). The two coding transcripts were then collaboratively reviewed, and a final set of themes generated.

\section{RESULTS}

The ethical themes that emerged from the stakeholder meeting are described in table 2 . The three primary themes include: (1) beneficence and non-maleficence; (2) autonomy and (3) distributive justice. The themes and their subthemes are discussed ahead, accompanying quotations from the meeting are available in the online supplemental material.

\section{Beneficence and non-maleficence \\ Patient benefits}

Patient-reported benefits centred on the perceived convenience offered by the ISBCS protocol. Patients reported that the fewer hospital visits, due to one pre-op assessment and one operation attendance, made the process convenient. The benefit of one recovery period permitted patients to return to prior commitments, such as work and caring responsibilities, sooner than the traditional protocol of delayed sequential bilateral cataract surgery (DSBCS).

Non-ophthalmologist health professionals reported issues with the traditional protocol that could be addressed by ISBCS. Nurses working with post-DSBCS surgical patients reported anisometropia to be an issue that was frequently observed. This had implications on a patient's quality of life during this period, and therefore was reported to directly factor in one nurse's decision to undergo ISBCS themselves. Ophthalmologists recognised the imposition of repeat hospital visits on patients' lives. Stakeholders recognised that repeat visits also had knock-on effects for patient's employment, or those acting as primary carers. One ophthalmologist highlighted how the geographical isolation of some patients amplified the direct patient benefits ISBCS offered.

\section{Patient risks}

The risks associated with ISBCS formed a central theme of the meeting. Discussions initially focused on the 'minor' complications following ISBCS. Specifically, the inability for the protocol to permit the power of the second lens to be modified based on 
the visual outcome of the first eye's surgery. Stakeholders agreed that large refractive errors were a rare occurrence following ISBCS, primarily due to technological advances in biometry accuracy. However, it was acknowledged that small refractive errors may still occur. Stakeholders highlighted that the shape of certain eyes predisposed patients to receiving an inaccurate lens power prediction. Therefore, this should be considered an exclusion criterion, as already stipulated in the National Institute for Health and Care Excellence (NICE) guidance. One ophthalmologist contended that the evidence base behind modifying the second lens power based on the visual outcome of the first eye was limited.

Stakeholders raised concerns surrounding how vulnerable populations would cope with minor complications that could arise following ISBCS. One complication specifically discussed was cystoid macular oedema (CMO), condition resulting in fluid build-up within the retina which may occur postcataract surgery. $\mathrm{CMO}$ and other minor complications, if occurring in in both eyes, could require two different treatment regimens of drops to resolve. The ability to successfully manage these treatments regimes and attend to usual activities of daily living with compromised vision could be an overwhelming task for some patients. Stakeholders felt that the ability of patients to self-manage minor complications should be a consideration by surgeons during the consent process.

Within the subtheme of 'patient risk' arose the duty of surgeons to minimise the harm to the patient. This principle was reflected by religious persons present and was emphasised by those representing Muslim and Catholic viewpoints. Stakeholders stipulated surgeons should only offer ISBCS if they had a good surgical track record, hospital safety record and adhered to accepted guidelines for the procedure. Medico-legal representatives also recommended doctors performing ISBCS adhered to NICE guidelines and maintained adequate exclusion criteria of high-risk cataract patients. However, it was acknowledged in certain situations it may be justifiable to deviate from the guidelines. Legal representatives highlighted that should a serious complication occur, there will likely be scrutiny of whether ISBCS was appropriate to have been offered for that case.

\section{The uncertainty of risk}

The uncertainty regarding the risk of severe sight-threatening complications, such as bilateral endophthalmitis, was discussed at the meeting. To estimate a risk of this occurrence, suggestions were made to analyse data obtained retrospectively from countries currently undertaking ISBCS. A data series following 100 000 patients undergoing ISBCS was described by one ophthalmologist. The results of this study found 12 patients experienced unilateral endophthalmitis, but there were no reports of bilateral infections. Stakeholders therefore stated that the risk of bilateral endophthalmitis is very low, provided that appropriate protocols are followed.

Due to the uncertainty associated with bilateral endophthalmitis, some religious members enquired to the possibility of designing future trials to fully quantify the risk. Ophthalmologists stated that as the incidence of severe complications was very low, designing appropriately large prospective trials to produce an accurate estimate is a difficult and expensive task. Debate surrounded how to accurately extrapolate the calculated risk of unilateral endophthalmitis, in order to produce an accurate estimate of the risk for bilateral endophthalmitis. One ophthalmologist treated them as independent variables, producing an estimate of the overall risk at 1 in 16 million. Another ophthalmologist did not agree with this approach, stating the factors that predisposed the patient to endophthalmitis in the first eye would also be present in the second.

\section{Patient interpretation of the risk-benefit analysis}

As there is an uncertainty associated with certain ISBCS complications, discussions turned to the importance of a risk-benefit analysis and how it should be undertaken. Stakeholders highlighted a desire that the risk-benefit analysis should be a patientcentred decision, as each individual would attribute different significance to each material (personal) risk and benefit associated with ISBCS. As the benefits and the risks of ISBCS are born directly by the patient, stakeholders felt ethically it should fall to the patient's decision to undertake the surgery.

Stakeholders acknowledged that the weighing of statistics relating to the risks and benefits for any given procedure is an inherently difficult task. This is exacerbated in the case of bilateral endophthalmitis, where the exact risk cannot be accurately quantified. Therefore, stakeholders stipulated that surgeons should ensure that patients understood the implications of any decision made, and this discussion should be fully documented within the notes.

Ethicists and religious persons among the group were keen to distinguish the difference between risk and hazard. Although stakeholders considered the risk of bilateral endophthalmitis to be lower than unilateral endophthalmitis, the hazard of both complications are not equal. Stakeholders stated the individual and societal effect of a patient being rendered completely blind is inherently a more hazardous outcome than that of unilateral blindness. Therefore, emphasis was placed on effectively articulating this risk to patients in a comprehensive way.

\section{Autonomy}

\section{Informed consent}

The stakeholders expressed a united consensus for patient centred decision making. An aspect of this included the oftennuanced process of informed consent. This concept was particularly emphasised by those attending in a healthcare professional capacity. Stakeholders emphasised the consent processes for ISBCS mirrored that of the single eye procedure. However, the uncertain risks associated with ISBCS does make consenting a more involved process. Viewpoints from those representing a religious perspective echoed the importance of gaining informed consent. Specific distinctions were made to ensure information was represented accurately and with integrity, to gain a truly 'informed' consent.

Medico-legal perspectives highlighted the law's view of obtaining informed consent. One lawyer stated they considered the signing of a consent form on the day of surgery to be primary evidence of professional misconduct, implying the patient had taken the decision before contemplating the risks of the procedure. The concept of consent following the landmark court ruling of Montgomery vs Lanarkshire Health Board was raised several times throughout the meeting. The ruling included the stipulation that a doctor must counsel patients on the advantages of disadvantages of any reasonable alternative procedures, including procedures not being offered.

\section{The barriers to communication}

Stakeholders, both professional and lay persons, recognised that there were barriers in gaining a truly informed consent. Providing adequate information to ensure patients understood the risks and benefits of ISBCS was considered essential to make an informed decision. Issues around informed consent primarily linked to the ability surgeons to communicate information 
adequately. Obtaining informed consent through satisfactorily communicating complex information was described as a generic problem across medicine. Stakeholders recognised the speed of patients to understand and weigh information is variable; patients should therefore be given adequate time to decide on options provided. This was compounded by the studies demonstrating that a patient's ability to understand and retain information in a stressful clinical environment was poor. Certain stakeholders also described how factors such as the cultural and educational backgrounds of their patients had to be taken into account when delivering information to ensure understanding. To tackle this, suggestions were made to modify the consent process to shift the burden of patient understanding out of the clinic, through the use of online applications designed to undertake the consenting process.

\section{Distributive justice}

The allocation of resources: the individual versus the collective

Stakeholders recognised that there was a disparity between the amount of resources a society spends on healthcare and the quality of healthcare the public wants delivered. Upholding distributive justice through conserving resources was described as one of the duties of a doctor. The health economist present discussed several studies that demonstrated a monetary cost saving for ISBCS. They concluded that based on current analysis, there was an estimated \$C500 of savings per patient when undertaking ISBCS compared with DSBCS. Disagreements arose over the strength of the current cost-benefit analysis available. One ophthalmologist stated a full economic analysis had not been completed, as the cost had not been offset against the financial effect of blinding a patient.

Some stakeholders suggested the potential economic savings to society that ISBCS offered was itself an important ethical consideration. These stakeholders recognised the importance of the ethical distribution of resources in an 'economic fixed-pot' such as the National Health Service (NHS) and felt there was a duty of doctors to produce the most efficient model possible. The proposed economic benefits should result in the procedure being offered more routinely to the appropriate patients. It was argued that although an initial small saving per operation $(\sim £ 300)$ is predicted, the potential quantity of operations carried out could result in $£ 30$ millions of savings per year. Stakeholders recognised that the economic savings would be applicable to the NHS as a whole and were unlikely to be directly used within ophthalmology.

Other stakeholders argued that although financial benefits of ISBCS may exist, it is currently not a robust enough analysis to be an ethically viable consideration. Religious and patient perspectives expressed the desire for the patient to remain at the centre of the consultation, with the doctor acting as an advocate for the patient's best interest but not for economic savings. Stakeholders agreed that the overall economics of the procedure should not be a conscious factor for clinicians when consulting patients on an individual basis. It was highlighted by the commissioner that should future economic analysis reveal there was a significant savings for ISBCS on top of the discussed patient benefits, ISBCS would become the default option for appropriate patients. Stakeholders subsequently concluded that currently the decision should be deferred to the patient's autonomy and their personal risk-benefit analysis of the procedure.

\section{DISCUSSION}

This meeting aimed to discuss the ethical challenges of the currently controversial procedure of ISBCS, as perceived by a group of 29 stakeholders. This meeting is unique, as there is currently no research that carefully evaluates the ethical considerations of ISBCS, beyond a risk-benefit analysis. ${ }^{37}$ The thematic analysis drawn from these data produced primary themes that echoed the previously described 'principles of bioethics' proposed by Beauchamp and Childress, these principles are considered non-hierarchical and in-tension with one another, therefore the utility exists in individuals balancing and weighing the prima faci duties within context specific scenarios. ${ }^{8}$ The ubiquitous application of these principles in education and professional guidance may explain the underlying utilisation of these principles by stakeholders. ${ }^{16}$ Maclin ${ }^{17}$ argues that even if not stated explicitly, these principles are invoked in ethical justifications within the medical field.

The first ethical principle discussed was the consideration of beneficence and non-maleficence. The benefits and risks of ISBCS discussed by stakeholders, were similar to those described in literature. ${ }^{34}$ Stakeholders felt the direct benefits of ISBCS were centred on patient convenience factors, and the ethically important risk revolved around the potential for bilateral endophthalmitis and bilateral vision loss. Stakeholders stated that the risk of bilateral endophthalmitis was very low and could not be accurately quantified based on existing data. This is in keeping with conclusions made from a systematic review by Kessel et al. ${ }^{7}$ Despite the low risk, stakeholders felt the complication of bilateral endophthalmitis remained an ethically important consideration, as the potential hazardous outcome of complete blindness was so severe. The highly emotive nature of binocular blindness, combined with the inevitability of an occurrence based on high cataract incidence, may explain why stakeholders attributed weight to this complication.

Due to the nature of the hazards associated with the protocol, stakeholders felt it was imperative that the patient is placed at the centre of the consultation, so they can be supported to make an individual decision based on their interpretation of the risk-benefit analysis. A patient-centred risk-benefit analysis acts to preserve the principle of autonomy through recognising patients are not a singular population, rather each has unique life situations, goals and expectations. ${ }^{18}$ Often discussed at the meeting, was the ruling on consent law undertaken in 2015 by the Supreme Court in the landmark case; Montgomery vs Lanarkshire Health Board. The ruling highlighted changes in law that had been evident in GMC guidelines previously, on the evolution of medicine from a paternalistic to a patientcentred approach. ${ }^{19}$ The Montgomery ruling stipulated that during the consent procedure a doctor must make the patient aware of any material risks of the intervention, where material risk is defined as: 'any risk to which a reasonable person in the patient's position would attach significance'. ${ }^{20}$ It could be argued the very low but theoretical potential for complete sight loss would always be a material risk to a 'reasonable patient', and therefore must always be disclosed.

The ethical importance of distributive justice factors was an area of debate at the meeting. The discussion illustrated the conflict between utilitarian and deontological approaches to the distribution of healthcare resources. Some ophthalmologist stakeholders viewed the dilemma from a utilitarian perspective. They sensed the financial cost-saving to society the ISBCS protocol provided was an important consideration, given the finite resources available within the NHS. This consideration is in contrast to literature that describes medical practitioners as primarily morally deontological in nature. ${ }^{21}$ The utilitarian perspective from stakeholders may have emerged from utilitarian-focused roles represented, coupled 
with the consideration of the strain ophthalmology services are under, which is argued to drive clinicians to consider utilitarian perspectives. ${ }^{21} 22$

In contrast, many patient and religious stakeholders focused on the deontological consideration of distributive justice. These stakeholders felt that clinicians considering the financial savings for ISBCS were no longer caring for the patient but caring for the system as a whole. Applying moral theory to resource allocation requires the reconciliation of the contrasting deontological and utilitarian perspectives. Within medicine, this is achieved by maintaining a deontological approach at the level of the patient-clinician interaction and considering the utilitarian perspectives at a 'higher level', such as through NICE committee evaluation. ${ }^{22}{ }^{23}$ Stakeholders reached a general agreement that distributive justice benefits of ISBCS should not be an active consideration for clinicians when offering ISBCS at the individual level. Instead, this should default to the previously discussed principles of patient autonomy and the patient's interpretation of the risk-benefit analysis.

At the meeting there were variable interpretations and debate within some of the elicited themes (particularly benefits, risk and justice), highlighting the interaction between the diversity of moral theory unique to each individual. However, one moral value held by stakeholders was undeniably firm and permeated across backgrounds. That morally, patient autonomy with a focus on informed consent must remain at the forefront of ISBCS, this consent must be authentically 'informed' due to the nature of the risks involved. This consensus is the product derived from the other ethical principles debated and reflects the 'in-tension' play of the bioethical principles. However, this consensus may simplify the intricacies of a surgeon-patient relationship and therefore satisfying this condition alone is not sufficient for ISBCS to be considered an ethical undertaking. An ethical surgeon must also dutifully apply patient safeguards, stringent protocols, demonstrate surgical competence and perform appropriate patient selection. The surgeon must meticulously adhere to accepted surgical protocols such as those published by the international society of bilateral cataract surgeons. ${ }^{24}$ All caveats must be satisfied for our ethical conclusion to inform clinical judgement.

\section{COVID-19 ADDENDUM}

Since the ethical meeting has taken place, the COVID-19 pandemic has resulted in profound ethical challenges across health and social care. In response to the pandemic, unprecedented reforms within the NHS have taken place including the widespread cancellation of elective operations and the rapid acceleration toward new technologies such as virtual consultations. This new world of 'minimal contact' healthcare may necessitate stakeholders to re-interpret the ethics of ISBCS in these evolving circumstances, both at an individual and population level. With the direct benefits of ISBCS centred on fewer visits to hospital, we may speculate how these factors would play into the way our stakeholders are constantly needing to adapt their risk-benefit analysis of the procedure. These new challenges add a further dimension to the complex ethical discussion of ISBCS.

\section{CONCLUSION}

This analysis provides a reference point for the ethical factors governing the often-controversial topic of ISBCS, for a select group of stakeholders. The stakeholders concluded offering ISBCS to be an ethical undertaking when patient autonomy was appropriately attained. This requires a patient's individual interpretation of the risk-benefit analysis, which must include an understanding of the low but unquantifiable risk of severe complications. Ethically, a surgeon must aim to minimise risks through the adaption of accepted surgical protocols and through performing appropriate patient selection. Based on current evidence, cost savings to healthcare that may occur following ISBCS may be considered a secondary benefit, whereas the primary benefit is centred on patient convenience factors. Stakeholders desired a future robust cost-analysis of ISBCS to take place within the UK health infrastructure.

Correction notice This paper has been updated since first published to amend author name 'Mehran Zarei-Ghanavati'.

Acknowledgements The authors would like to thank the stakeholder group who participated as non-author contributors in this ethical discussion. The authors would also like to thank the Royal College of Ophthalmologists for use of the venue.

Contributors MQ transcribed the meeting, performed qualitative analysis and took the lead in writing the manuscript. DG performed independent qualitative analysis. $\mathrm{CL}$ and JS devised the project, organised the stakeholder meeting and invited participants. JS attained funding for the project. CL and ASB wrote and reviewed the stakeholder questions, both participated in the stakeholder meeting. MZG provided specific ethical advice for input to the manuscript. Additionally, all authors provided significant contribution to the written work, rewriting sections where required with regular amendments to drafts. All authors approved the final version of the work.

Funding This study is funded by the National Institute for Health Research (NIHR) Programme for Applied Research grant RP-PG-0611-20013. The views expressed are those of the author(s) and not necessarily those of the NIHR or the Department of Health and Social Care.

Competing interests None declared.

Patient consent for publication Not required.

Provenance and peer review Not commissioned; externally peer reviewed.

Data availability statement All data relevant to the study are included in the article or uploaded as supplementary information.

Open access This is an open access article distributed in accordance with the Creative Commons Attribution Non Commercial (CC BY-NC 4.0) license, which permits others to distribute, remix, adapt, build upon this work non-commercially, and license their derivative works on different terms, provided the original work is properly cited, appropriate credit is given, any changes made indicated, and the use is non-commercial. See: http://creativecommons.org/licenses/by-nc/4.0/.

\section{ORCID iDs}

Matthew Quinn http://orcid.org/0000-0002-8877-5024

Mehran Zarei-Ghanavati http://orcid.org/0000-0002-0679-8055

Christopher Liu http://orcid.org/0000-0002-1045-196X

\section{REFERENCES}

1 The Royal College of Ophthalmologists. The way forward, 2015. Available: https:// www.rcophth.ac.uk/wp-content/uploads/2015/10/RCOphth-The-Way-Forward Cataract-300117.pdf [Accessed Feb 2020].

2 Singh R, Dohlman TH, Sun G. Immediately sequential bilateral cataract surgery: advantages and disadvantages. Curr Opin Ophthalmol 2017;28(1):81-6.

3 Grzybowski A, Wasinska-Borowiec W, Claoué C. Pros and cons of immediately sequential bilateral cataract surgery (ISBCS). Saudi J Ophthalmol 2016;30(4):244-9.

4 Chang DF. Simultaneous bilateral cataract surgery. Br J Ophthalmol 2003;87(3):253-4.

5 Lee E, Balasingam B, Mills EC, et al. A survey exploring ophthalmologists' attitudes and beliefs in performing Immediately Sequential Bilateral Cataract Surgery in the United Kingdom. BMC Ophthalmol 2020;20(1).

6 Buchan JC, Donachie PHJ, Cassels-Brown A, et al. The Royal College of Ophthalmologists' National Ophthalmology Database study of cataract surgery: Report 7, immediate sequential bilateral cataract surgery in the UK: Current practice and patient selection. Eye 2020;124.

7 Kessel L, Andresen J, Erngaard D, et al. Immediate sequential bilateral cataract surgery: a systematic review and meta-analysis. J Ophthalmol 2015;1.

8 Beauchamp TL, Childress JF. Principles of biomedical ethics. 6th edn. Oxford: Oxford University Press, UK, 2008

9 Taylor RM. Ethical principles and concepts in medicine. Handb Clin Neurol 2013:118:1-9. 
10 Gillon R. Ethics needs principles--four can encompass the rest--and respect for autonomy should be "first among equals". J Med Ethics 2003;29(5):307-12.

11 Ebbesen $M$, Jensen TG, Andersen $S$, et al. Ethical perspectives on RNA interference therapeutics. Int J Med Sci 2008;5(3):159-68.

12 Keskinbora KH, Keskinbora K. Ethical considerations on novel neuronal interfaces. Neurol Sci 2018;39(4):607-13.

13 Liu C. The ethics of immediately sequential bilateral cataract surgery Part 1. Available: https://www.youtube.com/watch?v=_Gnl8Y1RHGc [Accessed Feb 2020].

14 Liu C. The ethics of immediately sequential bilateral cataract surgery Part 2. Available: https://www.youtube.com/watch?v=y99nqLFrINQ [Accessed Feb 2020].

15 Braun V, Clarke V. Using thematic analysis in psychology. Qual Res Psychol 2006;3(2):77-101.

16 Page K. The four principles: can they be measured and do they predict ethical decision making? BMC Med Ethics 2012;13:10.

17 Macklin R. Can one do good medical ethics without principles? J Med Ethics 2015;41(1):75-8.

18 Ronen GM, Rosenbaum PL. Reflections on ethics and humanity in pediatric neurology: the value of recognizing ethical issues in common clinical practice. Curr Neurol Neurosci Rep 2017;17(5):39.
19 General Medical Council. Consent: patients and doctors making decisions together. GMC, 2008. Available: https://www.gmc-uk.org/-/media/documents/Consent English_0617.pdf_48903482.pdf [Accessed Feb 2020].

20 Montgomery (Appellant) V Lanarkshire health board (Respondent) (Scotland), 2015 Available: https://www.supremecourt.uk/decided-cases/docs/UKSC_2013_0136_ Judgment.pdf [Accessed Feb 2020].

21 Garbutt G, Davies P. Should the practice of medicine be a deontological or utilitarian enterprise? J Med Ethics 2011;37(5):267-70.

22 Conway P, Gawronski B. Deontological and utilitarian inclinations in moral decision making: a process dissociation approach. J Pers Soc Psychol 2013;104(2):216-35.

23 Mandal J, Ponnambath DK, Parija SC. Utilitarian and deontological ethics in medicine. Trop Parasitol 2016;6(1):5-7.

24 International Society of Bilateral Cataract Surgeons. ISBCS general principles for excellence in ISBCS 2009, 2009. Available: http://eyefoundationcanada.ca/wpcontent/uploads/2020/05/2010-09-01-FINAL-ISBCS-SBCS-suggestions-from-ESCRSBarcelona.pdf [Accessed Jul 2020]. 